\title{
EFECTOS DE UN PROGRAMA DE ACTIVIDAD FÍSICA INTENSA EN LA TENSIÓN ARTERIAL Y FRECUENCIA CARDIACA DE ADOLESCENTES DE 12-15 AÑOS
}

\section{EFFECTS OF AN INTENSE PHYSICAL ACTIVITY PROGRAM ON THE BLOOD PRESSURE AND HEART RATE OF 12-15-YEAR-OLD ADOLESCENTS}

\author{
Guillermo Felipe López Sánchez ${ }^{l}$ \\ Jonathan Nicolás López ${ }^{2}$ \\ Arturo Díaz Suárez ${ }^{3}$ \\ Facultad de Ciencias del Deporte, Universidad de Murcia, España \\ gfls@um.es
}

\begin{abstract}
Resumen
Introducción y objetivo: en la actualidad, las investigaciones encaminadas a mejorar la salud cardiovascular de las personas son de suma importancia. Este artículo se centra en estudiar los efectos de un programa de actividad física sobre la tensión arterial y frecuencia cardiaca de un grupo de adolescentes. Método: participaron 38 adolescentes (14 hombres y 24 mujeres), entre los 12 y los 15 años de edad (media de edad 13,61 y DE 1,00). Las variables estudiadas fueron: tensión arterial (sistólica y diastólica) y frecuencia cardiaca en reposo. La tensión arterial y la frecuencia cardiaca se midieron con el tensiómetro de brazo Visomat Comfort 20/40. El procedimiento ha sido: pre-test, intervención y pos-test. La intervención ha consistido en tres días a la semana de actividad física de alta intensidad, 15 minutos al día, durante 12 semanas. Resultados: se ha realizado un análisis de datos por medio del SPSS 22 y se han obtenido mejoras significativas en la tensión arterial y la frecuencia cardiaca (Sig. $<0.05$ ). Conclusiones: el programa de actividad física empleado en este estudio se presenta como una herramienta útil para mejorar la tensión arterial y la frecuencia cardiaca de adolescentes.
\end{abstract}

Palabras clave: Escolares, Educación Física, Salud Cardiovascular.

\begin{abstract}
Introduction and objective: research focused on the improvement of cardiovascular health is very important nowadays. This paper focuses on studying the effects of a physical activity program on the blood pressure and heart rate of a group of adolescents. Method: This investigation involved 38 adolescents (14 males and 24 females), ages between 12 and 15 years old (average age 13.61 and standard deviation 1.00). The variables considered were: blood pressure (systolic and diastolic) and resting heart rate. Blood pressure and heart rate were measured by a Visomat Comfort 20/40 arm sphygmomanometer. The procedure was as follows: pre-test, intervention and pos-test. The intervention consisted of 3 days per week of high-intensity physical activity, 15 minutes per day, during 12 weeks. Results: Data was analyzed through the SPSS 22 and significant improvements were obtained in blood pressure and heart rate $(\mathrm{Sig}<0.05)$. Conclusions: The physical activity program implemented in this study is a useful tool to improve blood pressure and heart rate in adolescents.
\end{abstract}

Keywords: Students, Physical Education, Cardiovascular Health. 


\section{Introducción}

La hipertensión arterial es uno de los principales factores que contribuyen al desarrollo de enfermedad cardiovascular, siendo mayor el riesgo si además se presenta junto al sobrepeso u obesidad (Álvarez et al., 2013). Aunque este problema es predominante en la edad adulta, no se debe menospreciar su presencia en la niñez y la adolescencia, edades donde se encuentra una incidencia que abarca desde el $2 \%$ al $13 \%$ (Rabelo, 2001).

Para prevenir este problema es necesario conocer y controlar los factores de riesgo asociados al desarrollo de hipertensión, interviniendo sobre aquellos que puedan ser modificables para producir mejoras en la salud cardiovascular. En la actualidad, muchos son los factores que incrementan el riesgo cardiovascular de la población infantil y adolescente, entre los que es posible encontrar una dieta inadecuada, cambios en las dinámicas familiares o el aumento del sedentarismo (Perichart-Perera, Balas-Nakash, Ortiz-Rodríguez, Morán-Zenteno, GuerreroOrtiz y Vadillo Ortega, 2008). Todos estos factores señalan la necesidad de investigaciones e intervenciones que procuren la mejora en la salud de esta población y la reducción del riesgo de enfermedades cardiovasculares, que puede encontrarse desde edades muy tempranas.

Durante la edad escolar y la adolescencia, la combinación de resultados de riesgo cardiovascular pueden estar asociados con la disfunción endotelial y principios del proceso aterogénico (Da Silva et al., 2014). Además, McEneny (2000) también señala que la hipertensión, junto con altos niveles de masa grasa, se asocia con enfermedades del corazón.

Asimismo, los adolescentes obesos presentan una disfunción autonómica cardiaca relacionada con una disminución de la actividad parasimpática y una reducida variabilidad de la frecuencia cardiaca (Farah, Ritti-Dias, Balagopal, Hill y Prado, 2012), además de presentar un aumento de la leptina, la resistencia a la insulina, el estrés oxidativo y la inflamación (López, López y Díaz, 2015f). A través de la actividad física, se puede conseguir mejorar mecanismos fisiológicos asociados, como la adaptación del sistema nervioso autónomo, la vasodilatación y la reducción de la rigidez arterial (Fagard, 2001; Harber et al., 2016).

El seguimiento de un estilo de vida activo, manteniendo una práctica regular de ejercicio físico y una dieta equilibrada aporta beneficios en niños y adolescentes, destacando la mejora del autoconcepto, la resistencia aeróbica, la fuerza muscular y el colesterol (Borrego et al. 2012, 2014, 2015a,b,c), así como mejoras en la composición corporal (López, Borrego y Díaz, 2013; López, López y Díaz, 2016e) y en la variabilidad de la frecuencia cardiaca (López et al, 2015f). Las adaptaciones producidas por la actividad física en poblaciones con necesidades especiales también son significativas, como en sujetos con Síndrome de Down (López y López, 2013) y escolares con Trastorno por Déficit de Atención por Hiperactividad -TDAH- (López et al. 2014, 2015a,b,c,d,e, 2016a,b), pudiendo mejorar parámetros saludables como la condición física, la imagen corporal, la frecuencia cardiaca, la presión arterial, la masa grasa, la coordinación dinámica general y segmentaria, la calidad del sueño y la calidad de vida. 
No obstante, y a pesar de sus múltiples beneficios, investigaciones recientes indican que los escolares no están realizando suficiente actividad física (López-Sánchez et al, 2016c,d; Pérez , Pfeffer, Rubio y Meléndez, 2011). Este hecho se traduce en un importante factor de riesgo para la población más joven, ya que lleva asociada la posible aparición de enfermedades cardiovasculares (Casado 2001). Por ello, es importante una concienciación de la población, junto con actuaciones tempranas y efectivas, puesto que la adolescencia se presenta como una etapa fundamental en la adquisición y consolidación de hábitos y estilos de vida (Chillón, Delgado, Tercedor y González, 2002).

Stevens,Cai, Evenson y Thomas (2002) también señalan que los niveles más bajos de actividad física, unidos a los niveles más altos de sedentarismo, se han asociado a un aumento del riesgo cardiometabólico global y el sobrepeso en los niños. Concretamente, en el parámetro de la tensión arterial, autores como Whelton, Chin, Xin y Jiang He (2002) sugieren ejercicio aeróbico para reducir la presión arterial en personas hipertensas y normotensas; no obstante, como señala el estudio de Chaves, Barbosa, Gonçalves y Guimarães (2007), hasta la fecha no existe un consenso sobre los valores normales de variación de la presión arterial por medio del ejercicio físico.

En este sentido, cabe destacar el estudio de López, López y Díaz (2015c), en el que se muestra cómo una intervención mediante actividad física de intensidad media-alta, con dos sesiones por semana de 60 minutos cada una, resultó insuficiente para producir mejoras significativas en la tensión arterial, frecuencia cardiaca de reposo y saturación de oxígeno en niños con TDAH.

De este modo, la intensidad del ejercicio puede ser un importante factor a tener en cuenta en la modificación de la tensión arterial. Datos recientes avalan que el entrenamiento de alta intensidad puede ser más eficaz para la mejora de la condición física y la función cardiaca en niños y adolescentes. Buchan et al. (2011), encuentra mejoras significativas $(\mathrm{P} \leq 0,05)$ en la presión arterial sistólica, la capacidad aeróbica y el índice de masa corporal (IMC) después de una intervención con un entrenamiento de alta intensidad. Los mecanismos fisiológicos que intervienen en la modificación de la tensión arterial, gracias al incremento de la intensidad de la actividad física, están conectados con la adaptación del sistema nervioso autónomo, la vasodilatación y la reducción de la rigidez arterial (Fagard, 2001; Harber et al., 2016).

Otra de las investigaciones que más información aporta a este respecto es la revisión llevada a cabo por Swain y Franklin (2010). En ella, se muestra cómo diversos estudios sostienen una mayor reducción del riesgo cardiovascular con la actividad física de intensidad vigorosa frente a la intensidad moderada, en parámetros como la presión arterial diastólica o la glucosa.

Atendiendo a los diferentes problemas detectados en investigaciones, como la falta de actividad física o la incidencia de la hipertensión arterial en niños y adolescentes (Álvarez et al., 2002), se hace necesario seguir investigando para lograr planteamientos efectivos, con la finalidad reducir el riesgo cardiovascular y mejorar la calidad de vida actual y futura de niños y adolescentes. Para ello, es importante continuar con la búsqueda de relaciones entre la actividad física y la tensión arterial, prestando atención al factor intensidad, cuyos datos aportados en 
investigaciones son aún escasos (Tanasescu et al., 2002), como posible medio para maximizar los beneficios obtenidos. Por tanto, debido a la relación directa entre actividad física y salud cardiovascular, son necesarias más intervenciones a través de actividad física. El objetivo de esta investigación es determinar los efectos producidos en la tensión arterial y la frecuencia cardiaca de adolescentes, tras una intervención mediante actividad física intensa de 12 semanas de duración. La hipótesis de partida es que, tras la intervención propuesta, se producirán mejoras significativas en la tensión arterial y en la frecuencia cardiaca de los escolares.

\section{Metodología}

\section{Participantes}

Un total de 38 adolescentes de 12 a 15 años (media de edad 13,61 y DE 1,00) participaron en el estudio. Los escolares pertenecían a la etapa educativa de Educación Secundaria Obligatoria (ESO). En función del sexo, había 14 hombres y 24 mujeres. En la Tabla 1 se describe la muestra con mayor detalle, en función de la edad y del sexo.

Tabla 1:

\begin{tabular}{cccc}
\multicolumn{5}{c}{ Descripción de la muestra, $N=38$} \\
\hline EDAD & $\begin{array}{c}\text { HOMBRES } \\
(\mathrm{n}=14)\end{array}$ & $\begin{array}{c}\text { MUJERES } \\
(\mathrm{n}=24)\end{array}$ & $\begin{array}{c}\text { AMBOS } \\
(\mathrm{n}=38)\end{array}$ \\
\hline 12 & 2 & 5 & 7 \\
13 & 2 & 6 & 8 \\
14 & 9 & 7 & 16 \\
15 & 1 & 6 & 7 \\
\hline
\end{tabular}

Esta investigación fue aprobada por la Comisión de Ética de Investigación de la Universidad de Murcia y todos los participantes firmaron un consentimiento informado.

El estudio fue cuasi experimental pre-post con un solo grupo de intervención, ya que el alumnado fue seleccionado por su interés en el programa de actividad física planteado, de forma no aleatoria, y se consideró como aspecto primordial de la investigación que todos los alumnos pudieran participar en el programa de actividad física.

\section{Procedimiento e instrumentos}

Los sujetos participantes en el estudio realizaron un pre-test, una intervención de 12 semanas y un pos-test. En el pre-test se analizaron las siguientes variables relacionadas con 
la salud cardiovascular de los escolares: tensión arterial sistólica, tensión arterial diastólica y frecuencia cardiaca en reposo. Durante la intervención, los escolares participantes realizaron tres veces a la semana sesiones de actividad física intensa de 15 minutos de duración. En el postest se volvió a analizar la tensión arterial y la frecuencia cardiaca en reposo de los escolares para comprobar si se habían producido mejoras respecto al pre-test.

La tensión arterial (sistólica y diastólica) y la frecuencia cardiaca en reposo (sentado) se midieron con el tensiómetro digital de brazo Visomat Comfort 20/40, el cual fue validado siguiendo el Protocolo Internacional de la Sociedad Europea de Hipertensión (Stergiou, Tzamouranis, Nasothimiou, y Protogerou, 2008). Se siguieron las directrices para realizar la medición de la tensión arterial del Grupo de Trabajo en Medición de la Tensión Arterial de la Sociedad Europea de Hipertensión (ESH Working Group on Blood Pressure Monitoring, 2008): los pacientes estaban relajados en posición sentada y con la espalda apoyada, el manguito a la altura del corazón, sin cruzar las piernas, en una habitación tranquila y silenciosa, con temperatura agradable, los sujetos descansaron al menos cinco minutos antes de realizar la medición y no hablaron antes o durante la medición.

Durante el tratamiento, los alumnos llevaron a cabo circuitos y ejercicios destinados a mejorar su salud cardiovascular. La actividad física fue intensa, la cual, según la OMS (Organización Mundial de la Salud), es aquella que requiere una gran cantidad de esfuerzo y provoca una respiración rápida y un aumento sustancial de la frecuencia cardíaca (aproximadamente $>6 \mathrm{MET}$ ). A la vez, en todo momento, se tenía en cuenta que se estaba trabajando con adolescentes y que para motivarlos se hacía necesario disfrazar las actividades propuestas mediante formas jugadas, alejándose de lo analítico y tradicional.

Todas las actividades se organizaban en espacios adaptados y con las reglas necesarias para conseguir que apareciesen sucesivas carreras y persecuciones cortas a intensidades altas. Ejemplos de actividades son: juegos competitivos, desplazamientos rápidos, footing... Se conseguía así una participación interválica a estas intensidades. Si se veía que la intensidad del juego disminuía, rápidamente se introducía una pequeña regla o modificación, o incluso los investigadores entraban a participar en el juego para conseguir mantener el ritmo de práctica.

Para valorar la intensidad de las sesiones de actividad física, estas fueron dirigidas por los investigadores, quienes observaban continuamente que los alumnos estuviesen trabajando a la intensidad adecuada y medían con regularidad la frecuencia cardiaca de los niños mediante pulsioxímetros de dedo OXYM2001.

\section{Análisis estadístico}

Se ha realizado un análisis estadístico por medio del Statistical Package for Social Sciences 22 (SPSS-22). En primer lugar, se ha aplicado la Prueba de normalidad de Shapiro-Wilk para determinar si las diferentes variables presentaban una distribución normal. A continuación, 
se ha realizado un contraste de igualdad de dos medias para muestras dependientes, para saber si se han producido diferencias significativas entre el pre-test y el pos-test. En las variables con distribución normal se ha utilizando la Prueba T-Student para muestras relacionadas (medidas repetidas), y en las demás variables, la Prueba de Wilcoxon (alternativa no paramétrica). El contraste de igualdad de dos medias para muestras dependientes se ha analizado en función de la muestra total y segmentando por sexo y edad.

\section{Resultados}

En la Tabla 2 se describe la comparación de medias de las diferentes variables en el pretest y el pos-test, indicando el grado de significatividad. En la Tabla 3 se vuelven a comparar las medias, segmentando en función del sexo. Por último, en la Tabla 4 se compara en función de la edad de los sujetos de la muestra.

Tabla 2.

Comparación de medias Pre-Post, $N=38$

\begin{tabular}{lllll}
\hline Variable & Media pre-test $(\mathrm{DE})$ & Media pos-test $(\mathrm{DE})$ & Dif. medias & Signific. \\
\hline TA sistólica & $124,50(13,14)$ & $116,08(11,94)$ & 8,42 & $0,000 * *$ \\
TA diastólica & $76,87(9,66)$ & $66,08(9,94)$ & 10,79 & $0,000 * *$ \\
FC & $75,76(11,48)$ & $73,26(11,61)$ & 2,50 & $0,002 * *$ \\
\hline${ }^{*} \mathrm{p}<0.05 * * \mathrm{p}<0.01$ & & & \\
\hline
\end{tabular}

En la Tabla 2 se observa que la tensión arterial (sistólica y diastólica) y la frecuencia cardiaca mejoraron de manera significativa en el pos-test, con un nivel de significación de $\mathrm{p}<0,001$ en los tres casos. En el Figura 1, se pueden apreciar estos resultados con mayor claridad.

Figura 1.

Comparación de medias Pre-Post, $N=38$

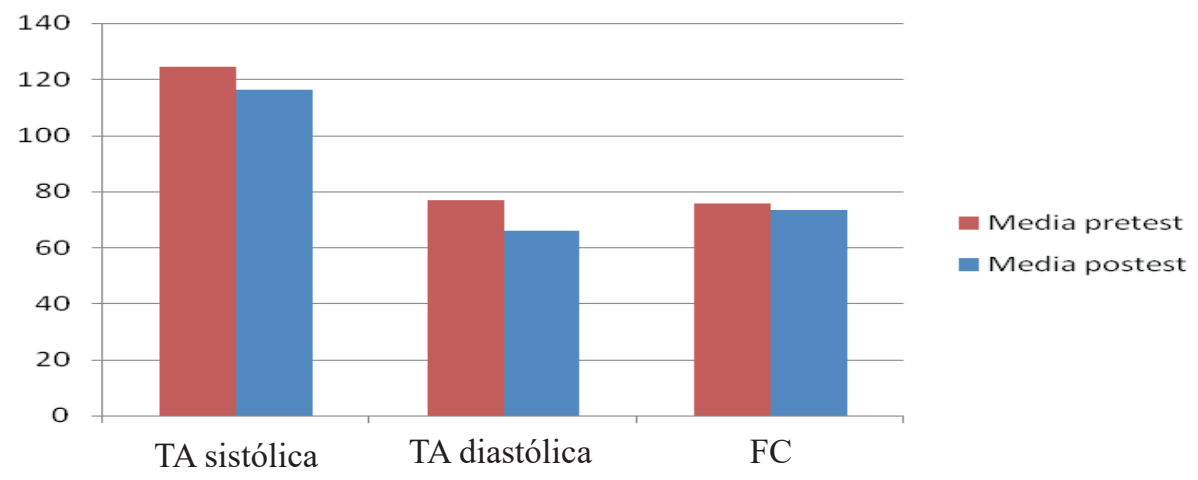


Tabla 3.

Comparación de medias Pre-Post, según sexo, $N=38$

\begin{tabular}{llllll}
\hline Variable & & Media pre-test (DE) & Media pos-test (DE) & Dif. medias & Signific. \\
\hline TA sistólica & Hombres & $128,07(15,60)$ & $122,93(15,74)$ & 5,14 & $0,025 *$ \\
& Mujeres & $122,42(11,31)$ & $112,08(6,63)$ & 10,34 & $0,000 * *$ \\
TA diastólica & Hombres & $78,00(8,31)$ & $70,00(9,58)$ & 8,00 & $0,000 * *$ \\
& Mujeres & $76,21(10,48)$ & $63,79(9,60)$ & 12,42 & $0,000 * *$ \\
FC & Hombres & $72,79(12,34)$ & $71,79(11,81)$ & 1,00 & 0,264 \\
& Mujeres & $77,50(10,83)$ & $74,13(11,67)$ & 3,37 & $0,003 * *$ \\
\hline$* \mathrm{p}<0.05 * * \mathrm{p}<0.01$ & & & & \\
\hline
\end{tabular}

Cuando se comparan las medias en función del sexo (Tabla 3), se puede observar que tanto en las mujeres como en los hombres se produjeron mejoras significativas en la tensión arterial sistólica y diastólica. Respecto a la frecuencia cardiaca, mejoró en ambos sexos, pero significativamente solo en las mujeres. Cabe destacar que la tensión arterial (sistólica y diastólica) fue mayor en los hombres, mientras que la frecuencia cardiaca fue más alta en las mujeres.

Tabla 4.

Comparación de medias Pre-Post, según edad, $N=38$

\begin{tabular}{llllll}
\hline Variable & & Media pre-test (DE) & Media pos-test (DE) & Dif. medias & Signific. \\
\hline TA sistólica & $12-13$ años & $120,60(9,61)$ & $112,20(6,00)$ & 8,40 & $0,005 * *$ \\
& $14-15$ años & $127,04(14,64)$ & $118,61(14,14)$ & 8,43 & $0,000 * *$ \\
TA diastólica & $12-13$ años & $74,80(8,38)$ & $62,60(7,38)$ & 12,20 & $0,000 * *$ \\
& $14-15$ años & $78,22(10,36)$ & $68,35(10,85)$ & 9,87 & $0,000 * *$ \\
FC & $12-13$ años & $81,13(12,17)$ & $78,47(13,07)$ & 2,66 & 0,115 \\
& $14-15$ años & $72,26(9,74)$ & $69,87(9,36)$ & 2,39 & $0,007 * *$ \\
\hline
\end{tabular}

$* \mathrm{p}<0.05 * * \mathrm{p}<0.01$

En función de la edad (Tabla 4), se pueden analizar los resultados por ciclo, de tal forma que los alumnos de 12-13 años pertenecen al primer ciclo de ESO y los de 14-15 años al segundo ciclo de ESO. Se observa que se produjeron mejoras significativas en ambos ciclos en la tensión arterial sistólica y diastólica. Respecto a la frecuencia cardiaca, mejoró en ambos ciclos, pero significativamente solo en segundo ciclo.

Es importante resaltar que los adolescentes de segundo ciclo de ESO presentaron niveles más elevados de tensión arterial que los de primer ciclo, observándose un aumento de la tensión 
arterial al aumentar la edad. En cambio, la frecuencia cardiaca fue mayor en los de primer ciclo, observándose una reducción de la frecuencia cardiaca al aumentar la edad.

\section{Discusión}

Los resultados obtenidos en el presente estudio mostraron cómo el programa de actividad física empleado ha producido una mejora significativa en la tensión arterial sistólica, diastólica y en la frecuencia cardiaca de los adolescentes. Estos datos se pueden comparar con los de otras investigaciones que también han estudiado el efecto de la actividad física en la tensión arterial y frecuencia cardiaca de escolares. La mayoría de estudios encuentran que la actividad física mejora la tensión arterial y la frecuencia cardiaca en ambos sexos y en diferentes edades, aunque algunos estudios no encuentran relaciones significativas.

En cuanto a la presión arterial sistólica, Perichart-Perera et al. (2008) observaron mejoras significativas, reduciéndose en un 63,9\% de los escolares, con una disminución significativa de $-4,79 \mathrm{mmHg}$ en niños y $-2,97 \mathrm{mmHg}$ en niñas $(\mathrm{p}=0.000)$, excepto en el grupo de 10 años. Dicho estudio contó con una muestra de 360 escolares (191 niñas y 169 niños), con edades comprendidas entre los 8 y los 14 años, que participaron en un programa de 20 minutos de actividad física realizado cinco veces a la semana durante 16 semanas. Los resultados son similares a los del presente estudio, en el que se produjo una mejora significativa en la tensión arterial sistólica en el pos-test $(\mathrm{P}<0,000)$.

En el estudio de López, López y Díaz (2014), el cual contó con una muestra final de 12 escolares con TDAH de sexo masculino y con un rango de 7 a 12 años, se realizó un programa de dos sesiones de actividad física aeróbica de media intensidad a la semana mediante circuitos y ejercicios para la mejora de la condición física durante 60 minutos al día. En este caso, los sujetos mejoraron los valores de tensión arterial sistólica y diastólica, aunque solo mejoró significativamente la frecuencia cardiaca tras el ejercicio $(p=0.029)$.

Álvarez et al. (2013) también encontraron diferencias significativas en la presión arterial sistólica de niños, adolescentes y adultos, reduciéndose en todos los casos tras la intervención $(\mathrm{p}=0.0001)$. En el caso de la diastólica, también se redujo en los tres casos pero solo de forma significativa en el grupo de adolescentes $(p=0.002)$ y adultos $(p=0.0001)$. En dicho estudio el grupo de niños estaba formado por 13 sujetos (6 niñas y 7 niños), el grupo de adolescentes contaba con 13 sujetos (6 niñas y 7 niños) y el grupo de adultos estaba formado por 55 sujetos (25 mujeres y 30 hombres). La intervención consistió en 60 minutos de ejercicio aeróbico en dos trayectos de $6 \mathrm{~km}$ en bicicleta a una velocidad $\leq 15 \mathrm{~km} / \mathrm{h}$.

El presente estudio también avala los resultados obtenidos por Reckziegel, Burgos, Reuter y Junior (2010), donde se analizaron a 230 escolares (107 niños y 123 niñas) con edades comprendidas entre los 7 y los 18 años, apuntando que la hipertensión presenta una relación con la baja actividad física y el alto índice de masa corporal. 
En la misma línea se encuadran los datos obtenidos en el estudio de Farah et al. (2012), donde 19 adolescentes participaron en un programa de seis meses, constituyendo un grupo de entrenamiento de alta intensidad (4 hombres y 5 mujeres) y otro grupo de baja intensidad (5 hombres y 5 mujeres). Los resultados mostraron cómo tras los seis meses de entrenamiento se disminuyó la presión arterial sistólica y diastólica $(\mathrm{p}<0.05)$ en ambos grupos, mientras que el grupo de entrenamiento de alta intensidad también consiguió otros beneficios adicionales como la reducción de la circunferencia de la cintura o la variabilidad de la frecuencia cardiaca $(\mathrm{p}<0.05)$. En relación con la Tabla 2, y en concordancia con los datos anteriores, el presente estudio mostró una mejora significativa en la tensión arterial sistólica en el pos-test $(\mathrm{P}<0,000)$.

Por otro lado, Salem, AboElAsrar, Elbarbary, ElHilaly y Refaat (2010) realizaron un estudio con 169 pacientes diabéticos tipo 1 (75 varones y 121 mujeres), con una edad comprendida entre los 12 y los 18 años, dividiéndolos en tres grupos que realizaron 1 día, 3 días o ningún día a la semana de actividad física. Sin embargo, en este caso la reducción de la presión arterial fue no significativa estadísticamente aparte de la presión arterial diastólica en el grupo que realizó tres días de actividad física por semana $(\mathrm{p}=0.04)$.

Krustrup et al. (2014) llevaron a cabo un estudio con 97 niños de 9 a 10 años (divididos en grupos de práctica y control) que realizaron, adicionalmente a su actividad normal, tres sesiones a la semana de 40 minutos de fútbol. En dicho estudio no se mostraron cambios en la frecuencia cardiaca en reposo ni en la presión arterial.

Diversos estudios respaldan que la actividad física de alta intensidad puede producir mayores mejoras en la tensión arterial que la actividad física de intensidad moderada, consiguiendo efectos más positivos sobre la presión arterial central y la rigidez arterial periférica (Fagard, 2001; Harber et al., 2016).

En relación con las tablas 2 y 3 del presente estudio, existen similitudes con el análisis de Chaves, Barbosa, Gonçalves y Guimarães (2007), en el cual se observó la respuesta de la presión arterial tras una prueba de esfuerzo de 218 adolescentes (139 hombres y 79 mujeres), con edades comprendidas entre los 10 y 19 años de edad, mostrando un mayor valor de presión arterial sistólica en los hombres, al igual que un incremento de la presión arterial diastólica conforme aumenta la edad.

Väistö et al. (2014), también mostraron, en una población de 468 niños con edades comprendidas entre los 6 y 8 años de edad, unos niveles de presión arterial sistólica y diastólica más bajos en las mujeres, $p=0.418$ y $p=0.629$, respectivamente. De forma similar, el análisis de Shahraki et al. (2011) reveló cómo la actividad física es capaz de reducir la presión arterial y la frecuencia cardiaca en un grupo de estudiantes atletas femeninas con una edad comprendida entre 21 y 23 años.

\section{Conclusiones}

Tras la intervención, se han encontrado mejoras significativas en la tensión arterial (sistólica y diastólica) y en la frecuencia cardiaca de la población en estudio, confirmando la hipótesis de 
partida. Estas mejoras han sido significativas en ambos sexos, salvo en la frecuencia cardiaca de los hombres. Asimismo, en función de la edad, las mejoras han sido significativas en los escolares de primer ciclo y en los de segundo ciclo, salvo en la frecuencia cardiaca de los de primer ciclo.

Según la muestra estudiada, la tensión arterial (sistólica y diastólica) es mayor en los hombres, mientras que la frecuencia cardiaca es más alta en las mujeres. En función de la edad, los adolescentes de 14-15 años presentan niveles más elevados de tensión arterial que los de 12-13 años, observándose un aumento de la tensión arterial al aumentar la edad. En cambio, la frecuencia cardiaca es mayor en los de 12-13 años, observándose una reducción de la frecuencia cardiaca al aumentar la edad.

Los resultados de este estudio son de suma importancia para la salud pública ya que el programa de actividad física planteado en este estudio, por su eficacia, puede servir de modelo para aplicar en los centros educativos y conseguir así que los niños y adolescentes tengan una mejor salud cardiovascular.

Por último, las principales limitaciones de este estudio fueron el tamaño de la muestra y la duración de la intervención. En futuras investigaciones se recomienda llevar a cabo otros programas de intervención mediante actividad física con muestras más amplias y mayor duración. Asimismo, se aconseja la utilización de diferentes tipos e intensidades de actividad física y la ampliación del rango de edad de la muestra. De este modo, habrá más recursos disponibles para mejorar la salud cardiovascular de los escolares mediante actividad física y se conocerá la eficacia de dichos recursos.

\section{Referencias Bibliográficas}

Álvarez, C., Olivo, J., Robinson, O., Quintero, J., Carrasco, V., Ramírez-Campillo, R., Andrade, D.C, \& Martínez, C. (2013). Efectos de una sesión de ejercicio aeróbico en la presión arterial de niños, adolescentes y adultos sanos. Revista Médica de Chile, 141(11), 13631370. http://dx.doi.org/10.4067/S0034-98872013001100001

Álvarez, I., Barrenechea, M.C., Basso, I., Bernadá, M., Bonelli, S., Caggiani, M., Duhagón, P., Espósito, A., Farré, Y., Gambetta, J. C., Giachetto, G., García, E., Mayado, C., Miralles, M. V., Peluffo, C., Rébori, A., Torterolo, V., Suárez, A. \& Velazco, M. (2002). Hipertensión arterial en el niño y el adolescente. Archivos de Pediatría de Uruguay, 73(1), 26-31.

Borrego-Balsalobre, F. J., López-Sánchez, G. F. \& Díaz-Suárez, A. (2012). Physical condition influence in self-concept of a teens group of Alcantarilla town. Cuadernos de Psicología del Deporte, 12(S2), 57-62. https://doi.org/10.1016/j.sbspro.2014.04.320

Borrego-Balsalobre, F. J., López-Sánchez, G. F. \& Díaz-Suárez, A. (2014). Relationships between physical fitness and physical self-concept in Spanish adolescents. PROCEDIA: Social and Behavioral Sciences, 132(1), 343-350. http://dx.doi.org/10.1016/j.sbspro.2014.04.320 
Borrego-Balsalobre, F. J., López-Sánchez, G. F. \& Díaz-Suárez, A. (2015a). Effects of a vigorous physical activity program in the endurance of primary school children. ATHLOS: International Journal of Social Sciences of Physical Activity, Game and Sport, 8(8), 31-46.

Borrego-Balsalobre, F. J., López-Sánchez, G. F. \& Díaz-Suárez, A. (2015b). Effects of a vigorous physical activity program in the strength of primary schoolchildren. TRANCES: Revista de Transmisión del Conocimiento Educativo y de la Salud, 7(3), 387-406.

Borrego-Balsalobre, F. J., López-Sánchez, G. F. \& Díaz-Suárez, A. (2015c). Influence of a vigorous physical activity program on cholesterol level of Primary schoolchildren. $A G O N$ : International Journal of Sport Sciences, 5(2), 60-71.

Buchan, D. S., Ollis, S., Young, J. D., Thomas, N. E., Cooper, S. M., Tong, T. K. \& Baker, J. S. (2011). The effects of time and intensity of exercise on novel and established markers of CVD in adolescent youth. American Journal of Human Biology, 23(4), 517-526. https://doi. org/10.1002/ajhb.21166

Casado, G. (2001). Estudio sobre la inactividad física como factor de riesgo cardiovascular en niños: relación con la capacidad aeróbica mediante tests de laboratorio y de campo. Proyecto de Tesis Doctoral. Universidad de Granada.

Chaves-Becker, M., Barbosa-Silva, O., Gonçalves-Moreira, I.E. \& Guimarães Victor, E. (2006). Arterial blood pressure in adolescents during exercise stress testing. Arq Bras Cardiol, 88(3), 297-300.

Chillón, P., Delgado, M., Tercedor, P. \& González, M. (2002). Actividad físico-deportiva en escolares adolescentes. Retos: nuevas tendencias en educación física, deporte y recreación, 1(3), 5-12.

Da Silva, D. F., Bianchini, J. A. A., Antonini, V. D. S., Hermoso, D. A. M., Lopera, C. A., Pagan, B. G. M. \& Junior, N. N. (2014). Parasympathetic cardiac activity is associated with cardiorespiratory fitness in overweight and obese adolescents. Pediatric cardiology, 35(4), 684-690. https://doi.org/10.1007/s00246-013-0838-6

ESH Working Group on Blood Pressure Monitoring (2008). European Society of Hypertension guidelines for blood pressure monitoring at home: a summary report of the Second International Consensus Conference on Home Blood Pressure Monitoring. Journal of Hypertension, 26(8), 1505-1530. http://dx.doi.org/10.1097/HJH.0b013e328308da66

Fagard, R. H. (2001). Exercise characteristics and the blood pressure response to dynamic physical training. Medicine and science in sports and exercise, 33(6), 484-492. https://doi. org/10.1097/00005768-200106001-00018 
Farah, B.Q., Ritti-Dias, R.M., Balagopal, P.B., Hill, J.O. \& Prado, W.L. (2013) Does exercise intensity affect blood pressure and heart rate in obese adolescents? A 6-month multidisciplinary randomized intervention study. Pedriatic Obesity, 9(2), 111-120. http:// dx.doi.org/10.1111/j.2047-6310.2012.00145.x

Harber, M., Ivey, E., Snyder, E., Arvin, K., Furrow, J. \& Hayes, E. (2016). High Intensity Interval Exercise Acutely Reduces Central Blood Pressure and Peripheral Arterial Stiffness. Medicine and science in sports and exercise, 48(5 Suppl 1), 1016-1016. https:// doi.org/10.1249/01.mss.0000488057.87311.72

Krustrup, P., Hansen, P. R., Nielsen, C. M., Larsen, M. N., Randers, M. B., Manniche, V., Hansen, L., Dvorak, J. \& Bangsbo, J. (2014). Structural and functional cardiac adaptations to a 10 -week school-based football intervention for 9-10-year-old children. Scandinavian journal of medicine \& science in sports, 24(S1), 4-9. http://dx.doi.org/10.1111/sms.12277

López-Sánchez, L. \& López-Sánchez, G. F. (2013). Enseñanza del tenis para personas con síndrome Down. Una experiencia práctica. EmásF: Revista Digital de Educación Física, 4(20), 1-9.

López-Sánchez, G. F., Borrego-Balsalobre, F. J. \& Díaz-Suárez, A. (2013). Effects of a physical activity program on body composition of school children of 3-5 years. SPORTK: Revista Euroamericana de Ciencias del Deporte, 2(2), 41-44.

López-Sánchez, G. F., López-Sánchez, L. \& Díaz-Suárez, A. (2014). Effects of a physical activity program on the physical fitness of schoolchildren with ADHD. RICCAFD: Revista Iberoamericana de Ciencias de la Actividad Física y el Deporte, 3(3), 24-37.

López-Sánchez, G. F., López-Sánchez, L. \& Díaz-Suárez, A. (2015a). Trastorno por déficit de atención con hiperactividad (TDAH) y actividad física. EmásF: Revista Digital de Educación Física, 32, 1-13.

López-Sánchez, L., López-Sánchez, G. F. \& Díaz-Suárez, A. (2015b). Effects of a physical activity program on the body image of schoolchildren with ADHD. Cuadernos de Psicologia del Deporte, 15(2), 135-142. http://dx.doi.org/10.4321/S1578-84232015000200015

López-Sánchez, L., López-Sánchez, G. F. \& Díaz-Suárez, A. (2015c). Effects of a physical activity program on the heart rate, blood pressure and oxygen saturation of schoolchildren with ADHD. Revista electrónica actividad física y ciencias, 7(1), 1-24.

López-Sánchez, G. F., López-Sánchez, L. \& Díaz-Suárez, A. (2015d). Effects of a physical activity program on the body composition of schoolchildren with ADHD. KRONOS: Revista Científica de Actividad Física y Deporte, 14(2), 1-9. 
López-Sánchez, G. F., López-Sánchez, L. \& Díaz-Suárez, A. (2015e). Effects of a physical activity program on the life quality of schoolchildren with attention deficit hyperactivity disorder. AGON: International Journal of Sport Sciences, 5(2), 86-98.

López-Sánchez, G. F., López-Sánchez, L. \& Díaz-Suárez, A. (2015f). Body composition and heart rate variability: relations to age, sex, obesity and physical activity. SPORTK: Revista Euroamericana de Ciencias del Deporte, 4(2), 33-40.

López-Sánchez, G. F., López-Sánchez, L. \& Díaz-Suárez, A. (2016a). Effects of a physical activity program on the sleep quality of schoolchildren with ADHD. SPORTK: Revista Euroamericana de Ciencias del Deporte, 5(1), 19-26.

López-Sánchez, G. F., López-Sánchez, L. \& Díaz-Suárez, A. (2016b). Effects of a physical activity program on the general dynamic and segmentary coordination of boys with ADHD. Journal of Sport and Health Research, 8(2), 115-128.

López-Sánchez, G. F., Ahmed, D., Borrego-Balsalobre, F. J., López-Sánchez, L. \& Díaz-Suárez, A. (2016c). Level of habitual physical activity in 8-9 years old schoolchildren from Spain and India. MHSalud: Revista en Ciencias del Movimiento Humano y Salud, 12(2), 1-10.

López-Sánchez, G. F., González-Víllora, S. \& Díaz-Suárez, A. (2016d). Level of habitual physical activity in children and adolescents from the Region of Murcia (Spain). SpringerPlus, 5(1), 1-1. http://dx.doi.org/10.1186/s40064-016-2033-8

López-Sánchez, G. F., Nicolás-López, J. \& Díaz-Suárez, A. (2016e). Effects of a program of intense physical activity on the body composition of adolescents from Murcia. SPORT TK-Revista EuroAmericana de Ciencias del Deporte, 5(2), 83-88.

McEneny, J., O’Kane, M. J., Moles, K. W., McMaster, C., McMaster, D., Mercer, C. \& Young, I. S. (2000). Very low density lipoprotein subfractions in Type II diabetes mellitus: alterations in composition and susceptibility to oxidation. Diabetologia, 43(4), 485-493. https://doi.org/10.1007/s001250051333

Pérez, M., Pfeffer, F., Rubio, M. \& Meléndez, G. (2011). Association of physical activity in 9 and 10 years old Mexican children with their weight and body composition. The FASEB Journal, 25(1), 781-786.

Perichart-Perera, O., Balas-Nakash, M., Ortiz-Rodríguez, V., Morán-Zenteno, J. A., GuerreroOrtiz, J. L. \& Vadillo-Ortega, F. (2008). Programa para mejorar marcadores de riesgo cardiovascular en escolares mexicanos. Salud pública de México, 50(3), 218-226. http:// dx.doi.org/10.1590/S0036-36342008000300005

Rabelo, L. M. (2001). Fatores de risco para doença aterosclerótica na adolescência. $J$ Pediatr, 77(2), 153-164. https://doi.org/10.2223/JPED.303 
Reckziegel, M. B., Burgos, M. S., Reuter, C. P. \& Junior, B. B. (2010). Interacción del perfil antropométrico y la actividad física con la tensión arterial en niños y adolescentes. Archivos de medicina del deporte, 27(137), 191-200.

Salem, M. A., AboElAsrar, M. A., Elbarbary, N. S., ElHilaly, R. A. \& Refaat, Y. M. (2010). Is exercise a therapeutic tool for improvement of cardiovascular risk factors in adolescents with type 1 diabetes mellitus? A randomised controlled trial. Diabetology \& metabolic syndrome, 2(1), 1-1.Stergiou, G. S., Tzamouranis, D., Nasothimiou, E. G. \& Protogerou, A. D. (2008). Can an electronic device with a single cuff be accurate in a wide range of arm size? Validation of the Visomat Comfort 20/40 device for home blood pressure monitoring. Journal of Human Hypertension, 22(11), 796-800. http://dx.doi.org/10.1038/jhh.2008.70

Stevens, J., Cai, J., Evenson, K. R. \& Thomas, R. (2002). Fitness and fatness as predictors of mortality from all causes and from cardiovascular disease in men and women in the lipid research clinics study. American Journal of Epidemiology, 156(9), 832-841. https://doi. org/10.1093/aje/kwf114

Swain, D. P. \& Franklin, B. A. (2006). Comparison of cardioprotective benefits of vigorous versus moderate intensity aerobic exercise. The American journal of cardiology, 97(1), 141-147. https://doi.org/10.1016/j.amjcard.2005.07.130

Tanasescu, M., Leitzmann, M. F., Rimm, E. B., Willett, W. C., Stampfer, M. J. \& Hu, F. B. (2002). Exercise type and intensity in relation to coronary heart disease in men. Jama, 288(16), 1994-2000. https://doi.org/10.1001/jama.288.16.1994

Väistö, J., Eloranta, A. M., Viitasalo, A., Tompuri, T., Lintu, N., Karjalainen, P., Lampinen, E.K., Agren, J., Laaksonen, D.E., Lakka, H.M., Lindi, V. \& Lakka, T.A. (2014). Physical activity and sedentary behaviour in relation to cardiometabolic risk in children: cross-sectional findings from the Physical Activity and Nutrition in Children (PANIC) Study. International Journal of Behavioral Nutrition and Physical Activity, 11(55), 1-10. https:// doi.org/10.1186/1479-5868-11-55

Whelton, S. P., Chin, A., Xin, X., \& He, J. (2002). Effect of aerobic exercise on blood pressure: a meta-analysis of randomized, controlled trials. Annals of internal medicine, 136(7), 493503. https://doi.org/10.7326/0003-4819-136-7-200204020-00006 


\title{
Financiación: Fundación Séneca - Agencia de Ciencia y Tecnología de la Región de Murcia. Consejería de Industria, Turismo, Empresa e Innovación de la CARM.
}

\author{
Recepción: 10 de mayo del 2016
}

Corrección: 26 de setiembre del 2016

Aceptación: 4 de noviembre del 2016

Publicación: 30 de noviembre del 2016

1 Guillermo Felipe López Sánchez. Licenciado en Ciencias de la Actividad Física y del Deporte. Maestro (especialidad Educación Física). Máster Universitario en Investigación en Educación Física y Salud. Máster Universitario en Formación del Profesorado de Educación Secundaria Obligatoria y Bachillerato, Formación Profesional y Enseñanza de Idiomas (especialidad de Educación Física). Investigador del Grupo de investigación deporte, gestión y recreación (INGESPORT). Profesor de la Facultad de Ciencias del Deporte, Universidad de Murcia, España.

2 Jonathan Nicolás López. Técnico Superior en Animación y Actividades Físicas y Deportivas. Graduado en Ciencias de la Actividad Física y del Deporte. Colaborador del Grupo de investigación deporte, gestión y recreación (INGESPORT) de la Universidad de Murcia (España).

3 Arturo Díaz Suárez. Licenciado en Educación Física. Doctor en Ciencias de la Educación. Catedrático de Universidad y Decano de la Facultad de Ciencias del Deporte de la Universidad de Murcia. Investigador principal del Grupo de investigación deporte, gestión y recreación (INGESPORT) de la Universidad de Murcia (España). 